\title{
Post-Launch Analysis of the Deployment Dynamics of a Space Web Sounding Rocket Experiment
}

\author{
Huina $\mathrm{Mao}^{\mathrm{a}}$, Thomas Sinn ${ }^{\mathrm{b}}$, Massimiliano Vasile ${ }^{\mathrm{b}}$, Gunnar Tibert ${ }^{\mathrm{a}, *}$ \\ ${ }^{a}$ Department of Aeronautical and Vehicle Engineering, KTH Royal Institute of \\ Technology, Stockholm, Sweden \\ ${ }^{b}$ Advanced Space Concepts Laboratory, University of Strathclyde Engineering, Glasgow, \\ United Kingdom
}

\begin{abstract}
Lightweight deployable space webs have been proposed as platforms or frames for a construction of structures in space where centrifugal forces enable deployment and stabilization. The Suaineadh project was aimed to deploy a $2 \times 2 \mathrm{~m}^{2}$ space web by centrifugal forces in milli-gravity conditions and act as a test bed for the space web technology. Data from former sounding rocket experiments, ground tests and simulations were used to design the structure, folding pattern and control parameters. A developed control law and a reaction wheel were used to control the deployment. After ejection from the rocket, the web was deployed but entanglements occurred since the web did not start to deploy at the specified angular velocity. The deployment dynamics was reconstructed from the information recorded in inertial measurement units and cameras. The nonlinear torque of the motor used to drive the reaction wheel was calculated from the results. Simulations show that if the Suaineadh started to deploy at the specified angular velocity, the web would most likely have been deployed and stabilized in space by the motor, reaction wheel and controller used in the experiment.
\end{abstract}

Keywords:

Space web, Reaction wheel, Centrifugal deployment, Centrifugal stabilization

\footnotetext{
${ }^{*}$ Corresponding author

Email addresses: huina@kth.se (Huina Mao), thomas.sinn@strath.ac.uk (Thomas Sinn), massimiliano.vasile@strath.ac.uk (Massimiliano Vasile), tibert@kth.se (Gunnar Tibert)
} 


\section{Introduction}

Structurally flexible deployable space structures are studied due to their light weights and high packaging ratios. A space web is a flexible structure tensioned by thrusters or by centrifugal forces in a spinning assembly. These webs can act as lightweight platforms for construction of large structures in space [1-3]. The idea of using space webs originates from the Japanese "Furoshiki Satellite" [4-8], a large membrane structure. In a previous sounding rocket experiment, three corner satellites were released radially by separation springs from the center satellite. Thrust control was applied to prevent recoiling. However, the web entangled due to out-of-plane motions or end of deployment shocks. A more reliable control deployment is desirable [9-12].

Centrifugal deployment is a feasible technique to construct large structures in space. The attitude and the shape are controlled by corner satellites using centrifugal forces by rotating the central satellite. Centrifugal deployment has been widely studied since the 1960s [13]. For example, simple control methods are used to obtain a stable deployment [9], geometrical stiffness can be induced by centrifugal forces for lightweight, flexible material without requiring stiff members to maintain their shapes [14], possible lower cost thanks to no rigid frame, and ideally reliable automatic deployment in orbit with low power consumption [15]. Spin stabilization is one of several strategies for space webs. The rotational inertia dominating in the plane of the spinning satellite keeps the out-of-plane motions of the web small.

In the past years there have been attempts at launching lightweight solarsails. IKAROS [16], the world's first successful solar sail, was deployed by centrifugal forces. The main hardware components were the electrically controlled panels, central mechanism deployment module and sails. Normally, the centrifugal force deployment of a space web could be split into two steps $[3,16-18]$ : in the first step, booms or panels are extended slowly and quasistatically controlled by guide rollers or other mechanisms; in the second step, the web is extended to a final flat shape by the centrifugal force. However, rollers or other extending mechanisms may take up too much space for small deployable structures. A one-step deployment method was thus identified as a possible choice for future web deployment without complicate extending mechanisms $[11,12,19]$. This method can also be used as a backup when sails or webs fail to deploy by roller mechanisms or stored strain energy. 
Therefore, it is important for future spinning webs to establish a feasible technique for a one-step deployment.

The difficulties in deployment control in space are web recoiling and entanglement. To reduce the risk of entanglement, an adequate choice of folding pattern and a proper control of deployment speed of the web are required [10]. A star-like folding patterns is a promising candidate for centrifugal force deployment. Experiments are required to verify the viability of the proposed folding and deployment. However, the zero-g condition in space is difficult to simulate on ground tests for large scale web deployment [3, 20]. Computational simulations, e.g., the finite element method [11, 21], multi-particle models $[19,22]$ and analytical methods $[9,11,12,23]$ have been used to analyze the dynamics of centrifugal force deployment. Ground scaled model experiments $[20,22]$ are also used to compare with simulations. However, near zero-g experiments are still required to validate the one-step deployment by centrifugal forces.

A team from the University of Strathclyde (Glasgow, UK), the University of Glasgow (Glasgow, UK), KTH Royal Institute of Technology (Stockholm, Sweden) was formed in 2010 to deploy a space web, the Suaineadh experiment, in milli-gravity conditions as a test bed of the one-step deployment concept. It was supported by the Advanced Concepts Team of the European Space Agency (ACT/ESA), the Swedish National Space Board (SNSB), the German Space Agency (DLR) and the Swedish Space Corporation (SSC) through the Rocket Experiments for University Students (REXUS) programme. The aims of the experiment were to deploy and stabilize a space web by means of the centrifugal forces acting on the spinning assembly. The design of the mechanical, communication and electrical systems and some lessons learned from the project are described in [24-26].

Suaineadh was ejected from the nosecone of the REXUS-12 sounding rocket and was able to use 140 seconds of weightlessness by flying in a parabolic trajectory. Four small daughter sections were attached to four corners of a square web, Fig. 1, and released from the initial folding state from the central hub. An active control method was used by a reaction wheel with the feedback from an inertial measurement unit (IMU) in the center hub. Four other IMUs were installed in daughter sections in order to provide information of the motion. Operational data were accumulated visually via cameras and on-board sensors. Suaineadh was deployed at an altitude of approximate $80 \mathrm{~km}$ in March 2012. Unfortunately, it could not be located after the impact, but was luckily found by chance almost 18 months 
later in September 2013, close to the predicted position in the impact zone. All data could be recovered from the on-board memory.

The objective of this paper is to analyze the result from the Suaineadh experiment and evaluate if the simple control law was effective. Various effects, like the web coiling direction and angular velocities, are discussed. Numerical model, previously used in the design, was also used for results analysis. It is emphasized that the reconstruction of the deployment dynamics is done with the limited information provided by the IMUs and the onboard cameras. Results are extracted from video footage as the IMU fixed to the central hub did not work during launch and the moment of inertia of the hub was not measured due to budget and time constrints. We hope that our lessons reported here could provide useful information for future similar projects.

The remainder of this paper is outlined as follows. In Sec. 2, an overview of the Suaineadh experiment design is presented. Section 3 describes the analytical model for the web satellite. In Sec. 4, experiment and simulation results are presented and discussed. In Sec. 5, conclusions of the experiment are presented.

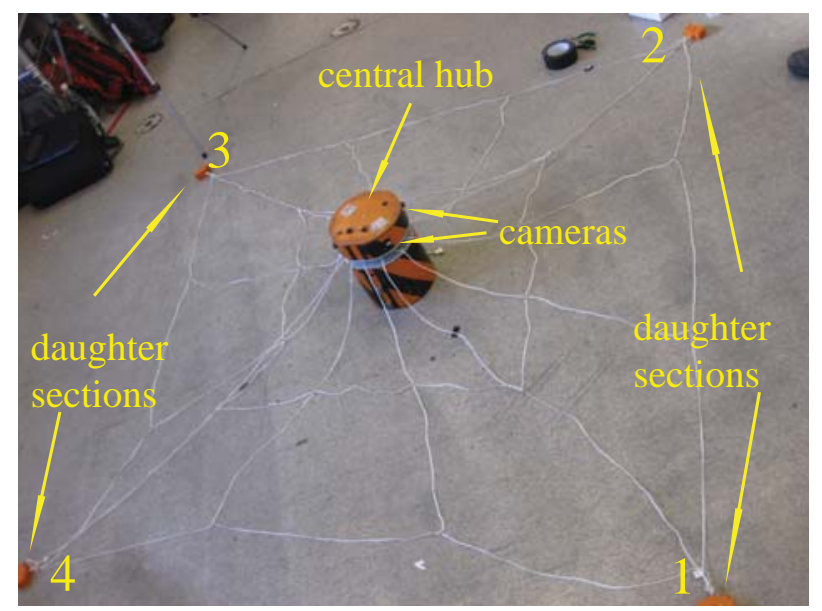

Fig. 1 Central hub and daughters (CHAD) with attached web at the launch campaign.

\section{Experiment Design}

The Suaineadh experiment could be divided into two distinct parts: (i) the central hub and (ii) the web with four corner daughter sections, Fig. 1. 
The central hub was responsible for controlling the deployment, stabilizing the web deployment and recording measurements. Four cameras on the central hub were used to record the web deployment and stabilization phases. The space web was folded around the central hub in a star folding pattern [12] and deployed by centrifugal forces acting on the attached daughters.

\subsection{Space web}

In the beginning of the project, the web was supposed to be a nonpermeable membrane. But the residual atmospheric drag at the ejection altitude would cause the membrane to fold out-of-plane according to finite element simulations. A membrane or even dense web would impair and decreased the significance of the acquired scientific data. Reasons for this were mainly because of the high speed, $500-600 \mathrm{~m} / \mathrm{s}$, of the central hub and daughters (CHAD) during deployment and the still significant air density around the apogee of the rocket trajectory. The REXUS-14 experiment Space Sailors [27] successfully deployed a solar sail on the top of the sounding rocket REXUS-14 at an altitude of $81 \mathrm{~km}$ in May 2013, but the sail collapsed under the aerodynamic pressure at the beginning of the descent as expected. In the end, a coarse web of braided Spectra cord was chosen, Fig. 1. This skeleton type web could overcome the problem of air pressure and still produce useful scientific data for post flight analysis.

\subsection{Spin direction of the hub and coiling direction of the web}

Figure 2(a) shows the REXUS-12 rocket spinning in a positive roll. The de-spinning mechanism of the rocket was intrinsically important to the control method used for the web deployment. If the experiment was mounted on a rocket which did not de-spin, assuming the angular velocity initially was $3-4 \mathrm{~Hz}$, the time span for full deployment would be too short to guarantee post-deployment stability, and a larger torque was required to transfer angular momentum from the reaction wheel to the hub. It could also be concluded from analysis that the hub started to recoil very early in the deployment process because the hub was rotating too fast.

After the rocket de-spun, the spinning frequency was almost $0 \mathrm{~Hz}$. Of course, if the web deployment would initiate at this speed, no web deployment would occur due to the lack of centrifugal forces acting on the daughter sections. Therefore the reaction wheel was used to spin up the CHAD before web deployment started. 
Before launch, the reaction wheel polymer bearings were preloaded with a very small force to eliminate play in the axial direction and the friction torque was minimised, Fig. 2(b). Due to gravity, the lower bearing was loaded slightly more than the upper one. During launch of the sounding rocket, an acceleration of around $20 \mathrm{~g}$ pushed the reaction wheel against the lower bearing and basically glued the wheel to the hub by friction. Hence, the wheel spun at the same rate as the rocket with an angular frequency of $3-4 \mathrm{~Hz}$ in positive roll. After thruster cutoff, the rocket de-spun to around $0 \mathrm{~Hz}$, but it was assumed that the reaction wheel was "weightless" due to the minimal friction torque from bearings. Thus the reaction wheel was basically decoupled from the hub and continued to spin at the same angular frequency as before thruster cutoff. This assumption was supposed to be verified by the sensor fixed on the reaction wheel, but that sensor failed to work in the experiment.

Considering that the reaction wheel motor could not reverse the spin direction because that would overheat the motor, it could only continue to increase in positive roll. According to the conservation of angular momentum, the hub would change its spin direction from positive roll, Fig. 2(b), to negative roll, Fig. 2(c). In other worlds, the reaction wheel reversed the spin direction of the hub before web deployment. Once the angular frequency of CHAD reached a specified initial angular velocity, the web started to deploy.

The coiling direction of the web was one of the important aspects of the web deployment. If it was coiled in negative roll direction as the spin direction of the hub, when the web started to release with a small initial angular velocity, Fig. 3, the web would be slack in the beginning, Fig. 3(b). Arms starting to recoil back in positive roll after being fully uncoiled, Fig. 3(c)-(d). The web might entangle due to the recoiling and thus fail to deploy. If the web was coiled in positive roll, Fig. 4(a), different to the spin direction of the hub, the web arms started to deploy and were extended straight by centrifugal forces and the arms rotated slower than the hub, Fig. 4(b). Without the reaction wheel, the rotation direction of the hub would change due to the conservational angular momentum, but the reaction wheel could prevent the web recoiling and keep it stable after full deployment, Fig. 4(c)-(d).

\subsection{Control method}

To prevent the web from getting into entanglement and to make the deployment stable, the centrifugal forces expand the web in the radial direction, $F_{c f}=m \omega^{2} R$, should be larger than the Coriolis force acting laterally, 


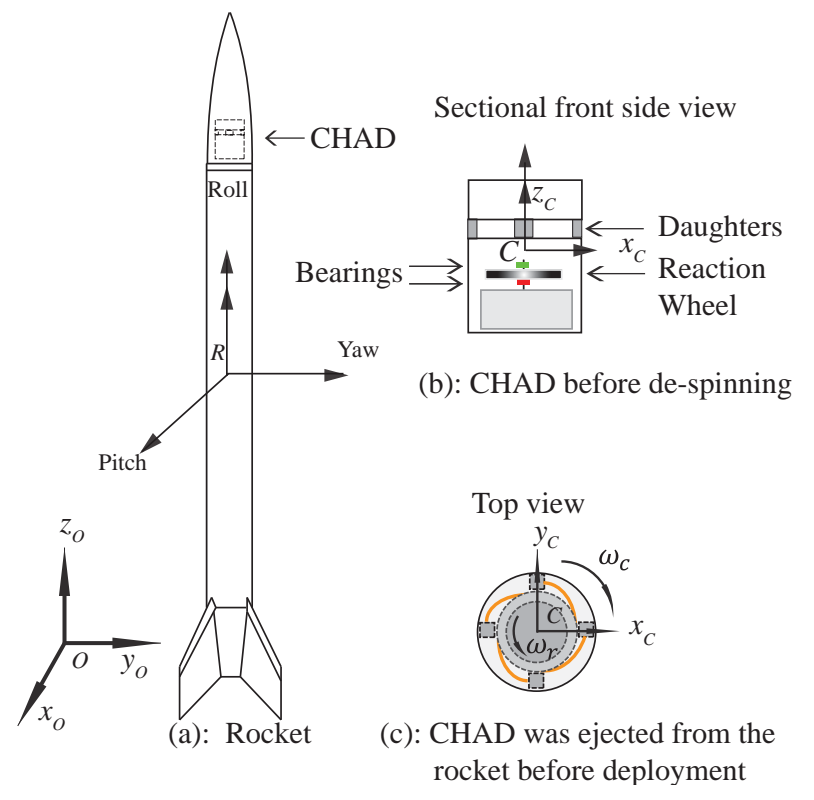

Fig. 2 (a) Model of the rocket; (b) CHAD was placed in the rocket before de-spinning; (c) CHAD was ejected from the rocket before deployment: the reaction wheel was accelerated in positive roll but changed the spin direction of CHAD to negative roll.

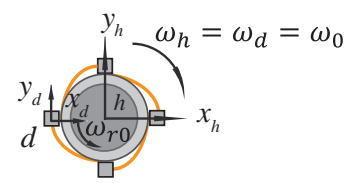

(a)

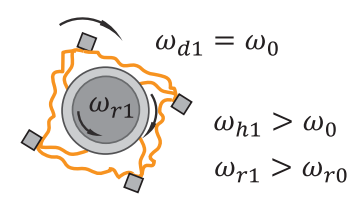

(b)

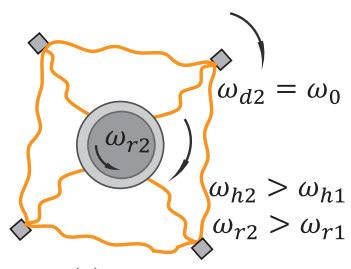

(c)

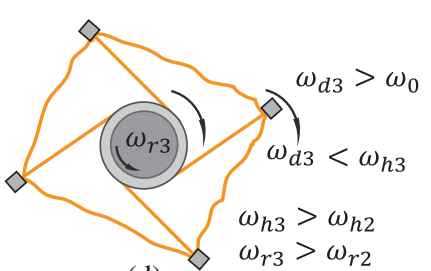

(d)

Fig. 3 Web arms were coiled in negative roll as the spin direction of the hub: (a), the web started to deploy; (b), web arms were slack in the beginning deployment; (c), web arms were fully deployed; (d) the web started to recoil back. 


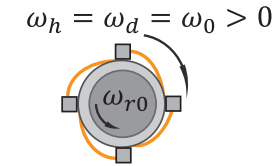

(a)
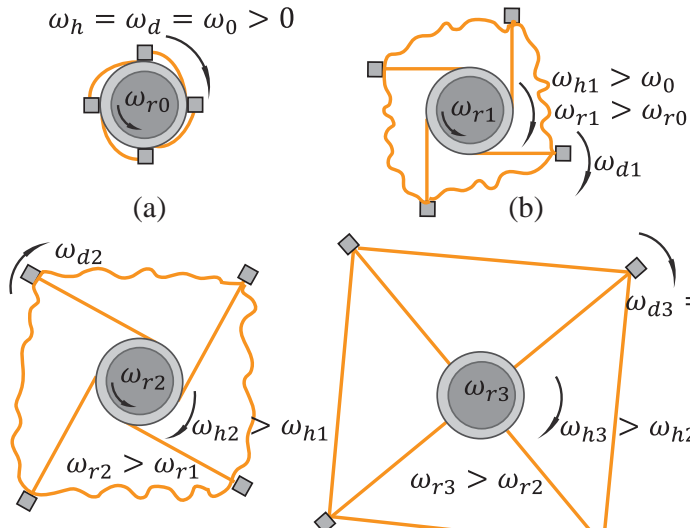

(c)

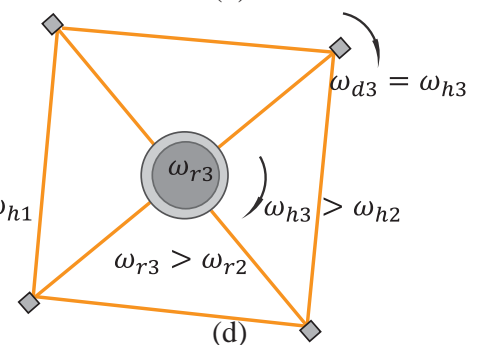

Fig. 4 Web arms were coiled in positive roll: (a), the web started to deploy; (b), web arms were extended in the beginning deployment; (c), web arms were fully deployed; (d) the web was fully deployed and stabilized by the reaction wheel.

$F_{c o}=2 m \dot{R} \omega$, and also larger than the inertial force, $F_{i n}=m \dot{\omega} R[9]$ :

$$
\begin{gathered}
\left|m \omega^{2} R\right| \gg|2 m \dot{R} \omega| \quad \text { or } \quad \gamma_{1}=\left|\frac{\omega R}{2 \dot{R}}\right| \gg 1 \\
\left|m \omega^{2} R\right| \gg|m \dot{\omega} R| \quad \text { or } \quad \gamma_{2}=\left|\frac{\omega^{2}}{\dot{\omega}}\right| \gg 1
\end{gathered}
$$

where $R$ is the deployment length of the web, $\omega$ is the angular velocity of the web.

Several control strategies of membranes deployment were studied in order to avoid re-coiling in one-step deployment [9, 11, 12]. A $20 \mathrm{~m}$ diameter membrane reflector was deployed at the end of a spacecraft using a one-step method during the Znamya-2 experiment in 1993 [9], demonstrated that a spin deployment of a gossamer structure can be controlled by simple means. The simple deployment process was driven solely by spinning up the stowed reflector using an on-board electric motor. A feedback control law with the angular velocity of the central hub was used to determine the control torque, $M_{h}$. Instead of the simple control law of Znamya-2, the "optimal MK law" [12], Eq. (3), was selected for the Suaineadh project:

$$
M_{h}=\max \left[0, \hat{M}_{h}\left(1-\frac{\omega_{h}}{\omega_{h f}}\right)\right]
$$


where $\omega_{h}$ is the angular velocity of the hub and $\hat{M}_{h}$ is the maximum input torque of the hub, $\omega_{h f}$ is the final angular velocity proposed in the experiment design. If $\omega_{h}$ could change direction, a small modification of Eq. (3) is needed. Assuming $\hat{M}_{h}$ and $\omega_{h f}$ have the same rotational direction, the "modified optimal MK control law" used in Suaineadh experiment could be expressed as:

$$
M_{h}=\hat{M}_{h} \cdot \max \left\{0, \min \left[1,\left(1-\frac{\omega_{h}}{\omega_{h f}}\right)\right]\right\}
$$

where the torque was provided by a reaction wheel,

$$
\begin{aligned}
& \hat{M}_{h}=-\hat{M}_{r} \\
& M_{h}=-M_{r}=-J_{r} \dot{\omega}_{r}
\end{aligned}
$$

where $\hat{M}_{r}, M_{r}, J_{r}$ and $\omega_{r}$ are respectively the maximum torque, applied torque, moment of inertia and angular velocity of the reaction wheel. The maximum angular acceleration of the reaction wheel was provided by a motor and depended on the angular velocity of the reaction wheel.

\subsection{Ground tests}

Ground tests were used to gain experience in the deployment dynamics as well as folding patterns. The issue of entanglement was the main concern since no mechanism in CHAD was responsible for recovering the web if entanglement occurred. If a membrane or dense web were used, entanglement could more easily be avoided, but a coarse web would have a higher risk of entanglement. For those reasons, ground tests utilizing a scaled down web of $0.6 \times 0.6 \mathrm{~m}^{2}$ were conducted on an air hockey table, Fig. 5 . The air hockey table had a very smooth surface and blowing air was used to counter the weight of daughters. The web was successfully deployed and the deployment could be divided into two phases: deployment phase, Fig. 5(a)-(d), and stabilisation phase, Fig. 5(e)-(f). The angular velocity was manually controlled. The central hub was slightly accelerated in phase 1 and a constant speed was kept in phase 2 .

The tests showed no collisions and entanglement between the daughters and implied that if the angular velocity of the hub was properly controlled by a reaction wheel in a milli-gravity environment, the web might be deployed without recoiling or entanglement. However, launch vibrations might change the folding pattern even if web was correctly coiled before launch because no mechanical barriers were used to ensure that the web did not move during the launch. 


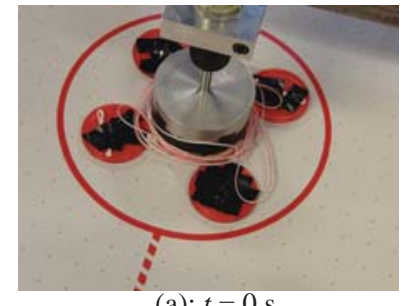

(a): $t=0 \mathrm{~s}$

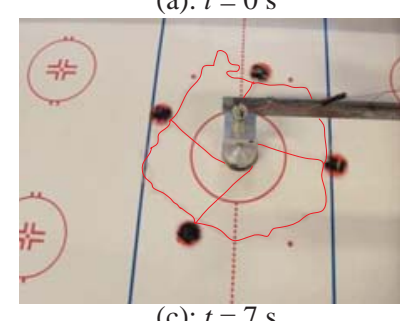

(c): $t=7 \mathrm{~s}$

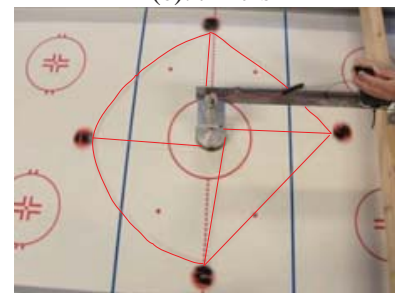

(e): $t=13 \mathrm{~s}$

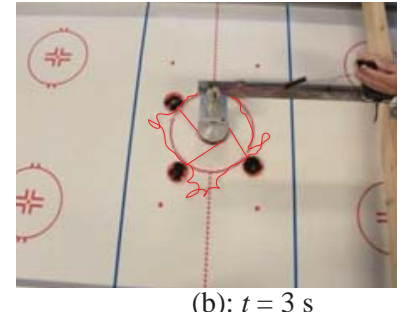

(b): $t=3 \mathrm{~s}$

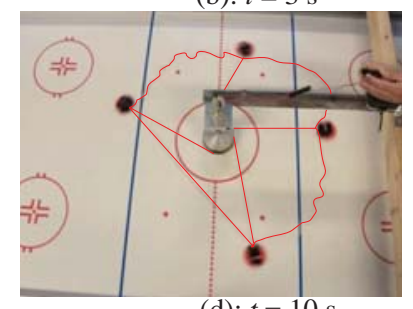

(d): $t=10 \mathrm{~s}$

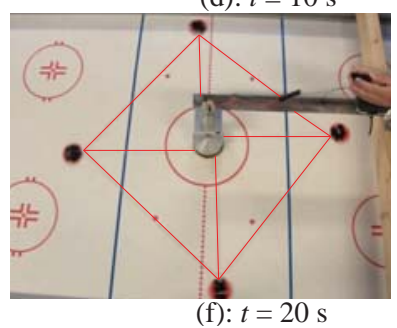

Fig. 5 Ground test of centrifugal force deployment of a scaled down $0.6 \times 0.6 \mathrm{~m}^{2}$ web on an air hockey table (the white web is highlighted by red lines for visibility). 


\section{Analytical Model for Simulations}

A simple analytical model with rigid bodies is proposed in $[9,11]$ for web deployment, Fig. 6. The web is folded into radial arms and is supposed to be deployed symmetrically and energy dissipation is neglected. Effects of the orbital forces and out-of-plane motions are not included.

\subsection{Equations of motion}

Three coordinate systems are required for the model in Fig. 6(a). Axis $\boldsymbol{e}_{z}^{(i)}$ is directed along the axis of rotation and $\boldsymbol{e}_{x}^{(i)}, \boldsymbol{e}_{y}^{(i)}$ are in the plane of deployment, where $i=0,1,2$. The first coordinate system is the inertial frame with its origin in the centre of the hub. The second coordinate system is the body frame rotated with an angular velocity. The third coordinate system is set on the attached point of a folded web arm. The CHAD rotates in negative roll, and the web is coiled in positive roll as discussed in Sec. 2.2.

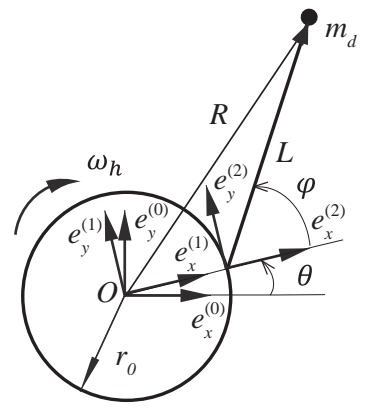

(a)

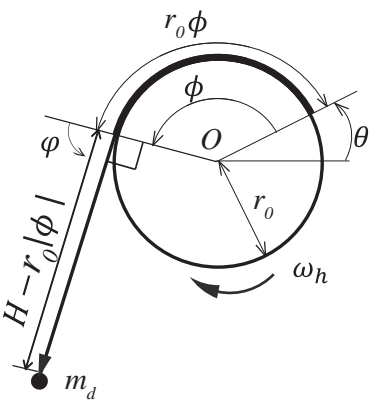

(b)

Fig. 6 CHAD rotates in negative roll and web arms are coiled in positive roll: (a) a deployed arm with a point mass in stability phase; (b) an arm coiled around the hub in deployment phase.

Equations (6) and (7) are respectively the equations of motion for stabilization and deployment phases [11], where $r_{0}$ is the radius of the hub, $J_{h}$ is the moment of inertia of the central hub around the axis of rotation, $n$ is the number of radial arms, $N$ is the tensile force in each arm, $L$ is the length of the web arm, $m_{w}$ is the total mass of the web, $\phi$ is the coiling angle of the 
arm, which equals to $H / r_{0}$ initially when the arm are completely coiled and $\varphi$ is the angle between $\boldsymbol{e}_{x}^{(2)}$ and the arm.

$$
\begin{array}{r}
J_{h} \dot{\omega}_{h}=M_{h}+n N r_{0} \sin \varphi \\
p\left(r_{0}\left(\omega_{h}^{2} \cos \varphi-\dot{\omega} \sin \varphi\right)-\ddot{L}\right)+q L\left(\omega_{h}+\dot{\varphi}\right)^{2}=N \\
p\left(r_{0}\left(\dot{\omega}_{h} \cos \varphi+\omega_{h}^{2} \sin \varphi\right)+2\left(\omega_{h}+\dot{\varphi}\right) \dot{L}\right)+q L\left(\dot{\omega}_{h}+\ddot{\varphi}\right)=0
\end{array}
$$

and

$$
\begin{array}{r}
J_{h} \dot{\omega}_{h}=M_{h}+s_{\phi} n N r_{0} \\
-s_{\phi} p r_{0} \dot{\omega}_{h}+q\left(H-r_{0}|\phi|\right)\left(\omega_{h}+\dot{\phi}\right)^{2}=N \\
s_{\phi} p r_{0}\left(\omega_{h}^{2}-\dot{\phi}^{2}\right)+q\left(H-r_{0}|\phi|\right)\left(\dot{\omega}_{h}+\ddot{\phi}\right)=0
\end{array}
$$

where

$$
\begin{aligned}
p & =m_{d}+\frac{m_{w} L^{2}}{n H^{2}} \\
q & =m_{d}+\frac{m_{w} L^{2}}{3 n H^{2}} \\
s_{\phi} & =\operatorname{sign}(\phi)
\end{aligned}
$$

\subsection{Initial and final angular velocity}

The magnitude of the initial angular velocity of the CHAD is very important for the deployment and stabilisation of the web. A high initial angular velocity decreases deployment time and the reaction wheel needs to provide a larger torque to the central hub to prevent recoiling. Also the final angular velocity should be large enough to provide sufficient out-of-plane geometrical stiffness. The final angular velocity depends on the maximum angular speed of the motor and the ratio between inertias. Therefore, proper initial and final angular velocities were determined before launch to ensure a stable deployment.

It was assumed that the final angular velocity $\omega_{f}=-1.9 \mathrm{rad} / \mathrm{s}$ and that the maximum torque of the motor could provide was constant. In order to simulate the angular velocity in the deployment, the moment of inertia of CHAD should be known. However, it has not be measured in the experiment. If the hub is a solid cylinder, the moments of inertia of the different parts 
are assumed as

$$
\begin{aligned}
J_{h} & =0.5 m_{h} r_{0}^{2} \\
J_{w} & =m_{w} r_{0}^{2} \\
J_{d} & =4 m_{d} r_{0}^{2} \\
J_{c} & =J_{h}+J_{w}+J_{d}
\end{aligned}
$$

where $J_{c}, J_{w}$ and $J_{d}$ are respectively the moment of inertia of CHAD, web and daughters with $m_{h}=6.1 \mathrm{~kg}, m_{d}=0.05 \mathrm{~kg}, m_{w}=0.0256 \mathrm{~kg}$ and $r_{0}=0.105 \mathrm{~m}$. Inserting them into Eq. (9), the moment of inertia of CHAD $J_{c}=36100 \mathrm{kgmm}^{2}$.

For the analytical model in Sec. 3.1, a 4th and 5th order Runge-Kutta method was used to simulate the deployment with different initial angular velocities in negative roll. $\hat{M}_{r}=11.8 \mathrm{mNm}$ and $\hat{M}_{r}=30 \mathrm{mNm}$ were selected in order to find a proper motor. Figure 7 shows how the deployment time depends on the initial angular velocities of the CHAD as well as the maximum torque of the motor. As the magnitude of the initial angular velocity increased, the influence of the motor decreased. For example, when $\omega_{0}=-1.256 \mathrm{rad} / \mathrm{s}$, the deployment time is almost the same for $\hat{M}_{r}=11.8 \mathrm{mNm}$ and $\hat{M}_{r}=30 \mathrm{mNm}$. Figure 8 shows that the hub will change its rotational direction in deployment when $\omega_{0}=-1.256 \mathrm{rad} / \mathrm{s}$ and $\hat{M}_{r}=11.8 \mathrm{mNm}$ because the motor cannot provide enough torque. This condition could be avoided by decreasing the initial angular velocity of the CHAD or increasing the motor torque capacity. Note that oscillations of the central hub is smaller when a more powerful motor is used. According to simulations, Fig. 8, a motor with $\hat{M}_{r}=11.8 \mathrm{mNm}$ can provide enough torque to the hub and stabilize the deployment within $100 \mathrm{~s}$ when the initial angular velocity of the hub is between -0.314 and $-0.628 \mathrm{rad} / \mathrm{s}$. A very high or a very low magnitude of initial angular velocity should thus be avoided. Following this analysis, $\omega_{0}=-0.628 \mathrm{rad} / \mathrm{s}(0.2 \mathrm{~Hz})$ was set as the proper specified initial angular velocity in our design.

\section{Results analysis}

\subsection{Experiment timeline}

Table 1 shows the design and actual timelines of the Suaineadh experiment. The timeline was designed based on GPS data from past REXUS missions. It was assumed that the rocket started to launch at $t=T+0 \mathrm{~s}$ 


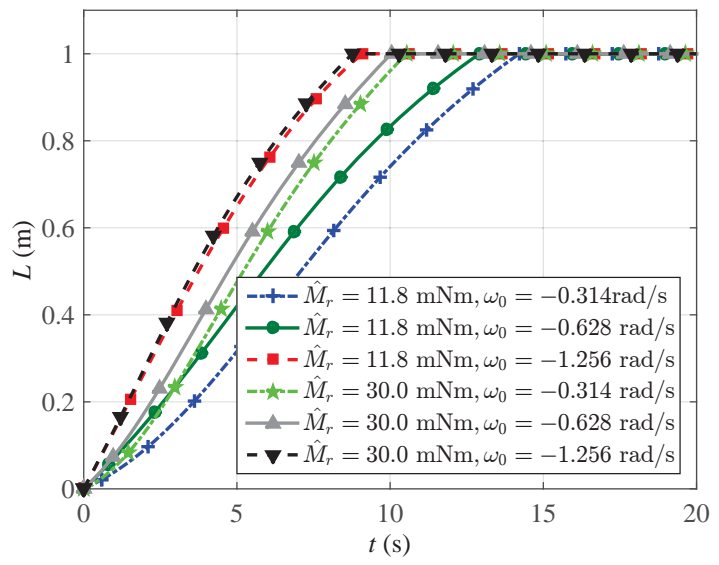

Fig. 7 Web deploys with different initial angular velocities and motors: deployed length of one arm.

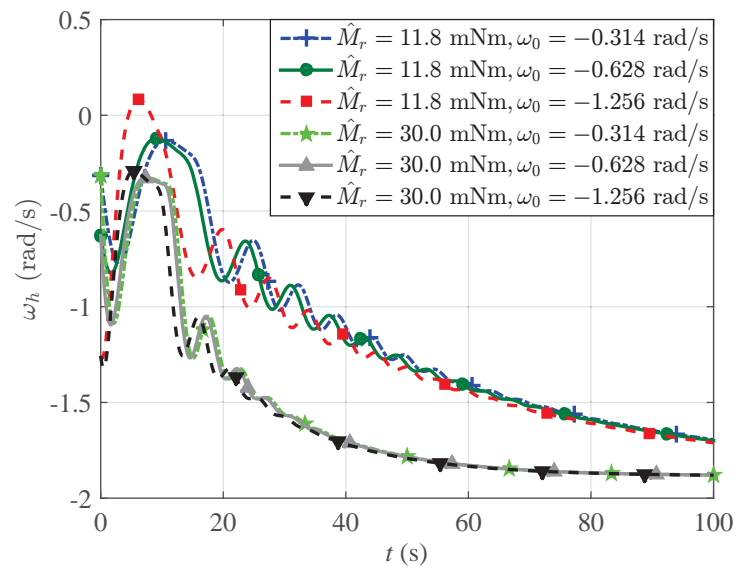

Fig. 8 Web deploys with different initial angular velocities and motors: angular velocity of the hub. 
and began to de-spin at $t=T+70 \mathrm{~s}$. At $t=T+80 \mathrm{~s}$, the CHAD was ejected from the nose cone of the rocket. When the distance between the rocket and the CHAD was sufficiently large and if the specified initial angular velocity was acquired after de-spinning, web deployment would be started. Otherwise, the reaction wheel would correct the angular velocity of the CHAD to the specified initial angular velocity. Assuming that the angular velocity of the CHAD after de-spinning was zero, the proper initial angular velocity was set to $-0.628 \mathrm{rad} / \mathrm{s}$ and the torque of the motor would provide a constant torque $M_{r}=\hat{M}_{r}=11.8 \mathrm{mNm}$ to accelerate the CHAD from 0 to $-0.628 \mathrm{rad} / \mathrm{s}$. According to the conservation of angular momentum before deployment,

$$
J_{c} \dot{\omega}_{c}=\hat{M}_{h}=-\hat{M}_{r}
$$

Assuming that the shape of the hub was a cylinder with a mass distribution between a shell and solid cylinder, the moment of inertia of the hub was

$$
0.5 m_{h} r_{0}^{2}<J_{h}<m_{h} r_{0}^{2}
$$

From Eqs. (9) and (11), the moment of inertia of CHAD, $J_{c}$, is between $36100 \mathrm{kgmm}^{2}$ and $69700 \mathrm{kgmm}^{2}$. Integrating Eq. (10) over time, a proper initial angular velocity could be reached between 1.9 and $3.7 \mathrm{~s}$. Thus, $3 \mathrm{~s}$ was assumed for the design in Table 1.

If the reaction wheel worked longer than $15 \mathrm{~s}$ before web deployment, a time backup mechanism would be activated. The daughter sections would be released after $17 \mathrm{~s}$, including $2 \mathrm{~s}$ for waiting between starting of data acquisition and releasing of daughter sections. Figure 9 shows that the web could be fully deployed within $13 \mathrm{~s}$ when the final angular velocity was $-1.9 \mathrm{rad} / \mathrm{s}$ and the system would become stable within $100 \mathrm{~s}$. As the maximum torque that could be provided by the reaction wheel is so small, the hub with the smallest inertia could be controlled better with respect to recoiling and stabilization. The deployment times were similar for the two inertias. Therefore, we expected the web to start deployment at $t=T+95 \mathrm{~s}$ and be fully deployed after $13 \mathrm{~s}$ and stabilized within $100 \mathrm{~s}$, Table 1.

The daughter release system should have been triggered as soon as the required spin rate was achieved. However, during the actual flight, the web was not deployed immediately. The time backup system was activated and the reaction wheel operated for $17 \mathrm{~s}$ before releasing daughters, Table 1. Such a long acceleration of the reaction wheel did not only reversed the spin direction of the CHAD but also provided a too high angular velocity of the 
CHAD. If the magnitude of initial angular velocity of the CHAD is far from the proper initial angular velocity, the deployment would be uncontrollable as previously discussed in Sec. 3.2. This unexpected deployment might be due to problems in the controller, sensors or programming, but the exact cause is still unclear. For example, the input value of $\omega_{h f}$ should be negative in design, but it might be written as a positive value in the programming of the controller. In actual flight, the angular velocity of the hub, $\omega_{h}$, was negative since it rotated around the negative roll. Therefore, the reaction wheel worked without daughters release as $\omega_{h}$ and $\omega_{h f}$ hade different directions in the "modified optimal MK control law", Eq. (4). Assuming that the angular velocity of the hub after de-spun was below the specified $\left|\omega_{h f}\right|$, the error could have been avoided by using absolute values in "modified optimal MK control law" as:

$$
M_{h}=\hat{M}_{h} \cdot \max \left\{0, \min \left[1,\left(1-\left|\frac{\omega_{h}}{\omega_{h f}}\right|\right)\right]\right\}
$$

Note that there was $2 \mathrm{~s}$ of waiting time between start of data acquisition and release of daughters, but the reaction wheel should not accelerate during those $2 \mathrm{~s}$ as otherwise it would increase the angular velocity of the hub beyond the specific initial angular velocity.

Table 1 Mission timeline

\begin{tabular}{lcc}
\hline \hline Event & Design time $(\mathrm{s})$ & Actual flight time (s) \\
\hline REXUS ignition & $T+0$ & $T+0$ \\
REXUS de-spin & $T+70$ & $T+70$ \\
CHAD ejection & $T+80$ & $T+80$ \\
Reaction wheel starts & $T+90$ & $T+90$ \\
Proper speed of the hub & $T+90+3$ & $T+90+15$ \\
Web deployment starts & $T+93+2$ & $T+105+2$ \\
Web is fully deployed & $T+95+13$ & $T+107+2$ \\
Web stabilization & $T+108+100$ & $T+109+168$ \\
\hline \hline
\end{tabular}

\subsection{IMUs and the motor}

There was one IMU fixed in each daughter section and one in the center to record accelerations and angular velocities. Unfortunately, the one fixed in the hub did not function properly. This might be one of the possible reasons 

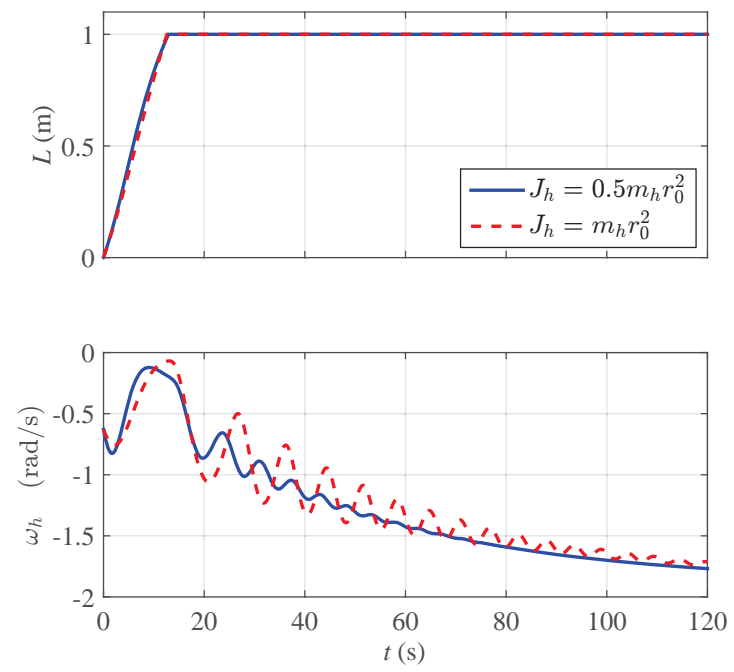

Fig. 9 Web deploys with different $J_{h}$ when $\omega_{0}=-0.628 \mathrm{rad} / \mathrm{s}, \omega_{f}=-1.9 \mathrm{rad} / \mathrm{s}$ and $\hat{M}_{r}=11.8 \mathrm{mNm}$.

why the web did not deploy at the proper specified initial angular velocity, as the angular velocity of the hub is an important parameter in our feedback control law, Eq. (4). In the following, we switch to angular frequency $f$ instead of angular velocity, where $f=\omega / 2 \pi$. Figure 10 shows the angular frequency of one IMU and the rocket. The cutoff range of the sensor in the rocket was $\pm 2.73 \mathrm{~Hz}$. The rocket rotated only around the roll axis. Before CHAD was ejected, the daughters and the rocket had the same acceleration and angular frequency. If the z-axis of the IMU was exactly parallel to the roll axis of the rocket, the angular frequency of $x$ - and $y$-axes were zero. Small values of rotational frequency around $x$ - and $y$-axes of the IMU was found in actual flight, Fig. 10, which might due to non-coinciding coordinate systems. However the data still can be used for the following analysis. For the IMUs of the daughters, the results were judged reliable and usable for our analysis, despite some noise and assembly offsets. The results from the IMU with the smallest noises is used in the following analysis.

After deployment, the path of each daughter was supposed to be calculated by integrating twice the acceleration of each IMU. However, due to digital and measurement noise, it was not possible to get the position of any IMU. For example, if the acceleration of the IMU in Fig. 11 was integrated 


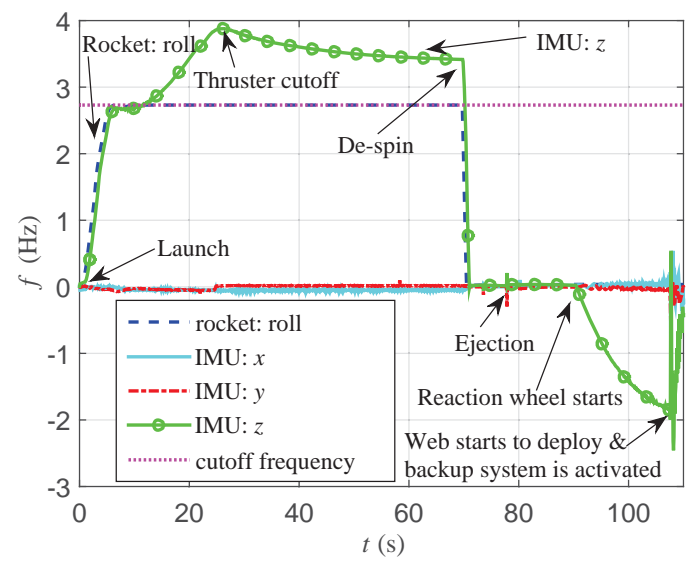

Fig. 10 Angular frequency of the rocket and one IMU in a daughter section before CHAD ejection.

twice, a small noise would lead to a huge difference in its path. Here the sensor's cutoff range of the IMU was $\pm 4 \mathrm{~g}$. The web deployed in $y$-direction with two peaks at $t=108.8 \mathrm{~s}$ and $t=113 \mathrm{~s}$, Fig. 11 . The first peak indicates that the daughter was fully deployed in less than $2 \mathrm{~s}$. The deployment time was too short compared with $13 \mathrm{~s}$ in design, Table 1, and it also implies that the initial angular frequency was too high before deployment. That would cause the deployment to be uncontrollable even if the reaction wheel worked properly. The second peak indicates the web was stopped recoiling by the hub. After this, the web started to deploy again. Similar acceleration peaks could also be found from other IMUs.

Figure 11 also shows that the accelerations of the IMU in $x$ - and $z$-axes were around zero after de-spinning. Milli-gravity conditions were given in the deployment and were kept during the whole deployment and stabilization phases.

\subsection{Moment of inertia of the hub and torque of the motor}

In order to better understand the deployment through simulations, a more accurate value of moment of inertia of the central hub and of the torque capacity of the motor are needed. Unfortunately, the exact $J_{h}$ was not measured in our experiment and the relationship between $\hat{M}_{r}$ and $\omega_{r}$ was unknown. Here we use the $17 \mathrm{~s}$ data of $f_{x}$ in Fig. 10 from when the reaction wheel started to work, $t=90 \mathrm{~s}$, to when the web started to deploy, $t=107 \mathrm{~s}$, to 


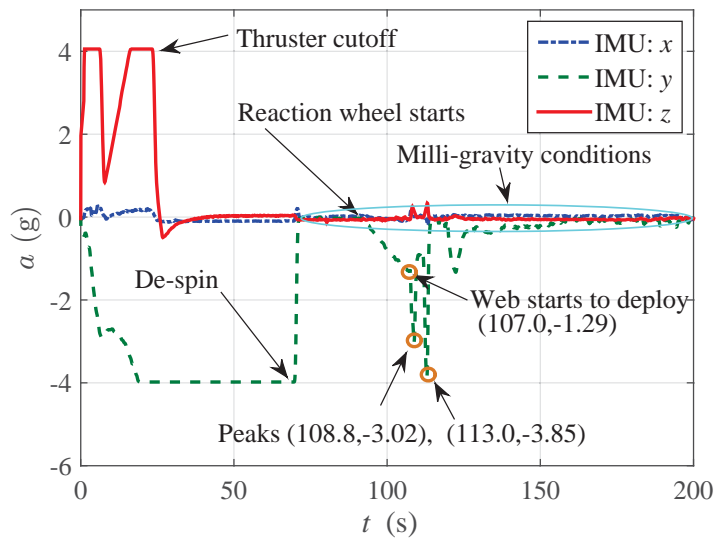

Fig. 11 Acceleration of one daughter IMU

calculate the approximate $J_{h}$ and $\hat{M}_{r}$. It was assumed that after de-spinning, the angular frequency of the reaction wheel was kept at the maximum spinning rate $3.88 \mathrm{~Hz}$ as before thruster cutoff. When the reaction wheel started to work, the angular frequency of the IMU in the $z$-direction changed from 0.02 to $-1.80 \mathrm{~Hz}$, Fig. 10. In this period, the reaction wheel worked with a maximum torque which nonlinearly depended on its angular frequency. Before deployment, the angular frequency of the CHAD was the same as the IMUs of the daughters. The equation of the changes in angular momentum of the CHAD around its axis of rotation (z-axis) is

$$
\begin{aligned}
J_{c} \dot{\omega}_{c}+J_{r} \dot{\omega}_{r} & =0 \\
J_{r} \dot{\omega}_{r} & =\hat{M}_{r}
\end{aligned}
$$

Before deployment, $J_{c}$ is a constant. Using sampled data, Eq. (13) can be expressed as

$$
\begin{aligned}
& J_{c}\left(\omega_{c_{i}}-\omega_{c_{i-1}}\right)=-J_{r}\left(\omega_{r_{i}}-\omega_{r_{i-1}}\right) \\
& J_{c}\left(\omega_{c_{i}}-\omega_{c_{i-1}}\right)=-\hat{M}_{r i}\left(t_{i}-t_{i-1}\right)
\end{aligned}
$$


then

$$
\begin{aligned}
\omega_{r_{i}} & =-\frac{J_{c}}{J_{r}}\left(\omega_{c_{i}}-\omega_{c_{i-1}}\right)+\omega_{r_{i-1}} \\
\hat{M}_{r i} & =-\frac{J_{c}}{\left(t_{i}-t_{i-1}\right)}\left(\omega_{c_{i}}-\omega_{c_{i-1}}\right)
\end{aligned}
$$

$i=1,2,3, \ldots, n$, where $n$ is the number of sampled data.

In the experiment, a Faulhaber motor 2232 BX4 (edition 2008) was used. The recommended maximum spin rate was $f_{\max }=175 \mathrm{~Hz}$ for a given ambient temperature of $22^{\circ} \mathrm{C}$ with $12 \mathrm{~V}$ input. It could provide more power with adequate cooling, for example, if the thermal resistance had a reduction of $55 \%$, the maximum spin rate could be more than $250 \mathrm{~Hz}$. In our experiment, the ambient environment temperature was very low and the maximum spin rate $f_{\max }=250 \mathrm{~Hz}$ was thus assumed.

Figure 12 shows the angular frequency of the reaction wheel, Eq. (15a), when different $J_{h}$ is considered for $J_{c}$ in Eq. (9). $f_{r}$ increases as $J_{h}$ increases because a higher $J_{h}$ needs a higher torque to reach the same angular frequency of the CHAD.

Figure 13 shows the maximum torque of the reaction wheel, Eq. (15b), which depends on the angular frequency of the reaction wheel. The stall torque of the motor is $M_{\text {stall }}=55.7 \mathrm{mNm}$. From Figs. 12-13, when $J_{h}$ was a little less than $0.6 m_{h} r_{0}^{2}$, the results satisfied the limitations of the angular frequency and stall torque.

The approximate $J_{h}$ can also be estimated based on geometry properties in Fig. 14. The total mass of the CHAD was $m_{c}=7.6 \mathrm{~kg}$. The inside structure without the reaction wheel had a mass $m_{\mathrm{in}}=3.9 \mathrm{~kg}$. The outside part of shell had a mass $m_{\text {out }}=2.2 \mathrm{~kg}$ which included four circular plates and two hollow cylinders, Fig. 14(b). It was assumed that the outside shell was assembled with the same material and thickness, and the central structure was evenly distributed. The masses of circular plates and hollow cylinders were

$$
\begin{aligned}
m_{\mathrm{cp}} & =\frac{4 m_{\mathrm{out}} \pi r_{0}^{2}}{4 \pi r_{0}^{2}+2 \pi r_{0}\left(h_{1}+h_{2}\right)} \\
m_{\mathrm{hc}} & =m_{\mathrm{out}}-m_{\mathrm{cp}}
\end{aligned}
$$

where $h_{1}=0.07 \mathrm{~m}$ and $h_{2}=0.18 \mathrm{~m}$, Fig. 14 . Thus $m_{c p}=1.0 \mathrm{~kg}$ and $m_{h c}=1.2 \mathrm{~kg}$ in Eq. (16). The moment of inertia of the CHAD without 


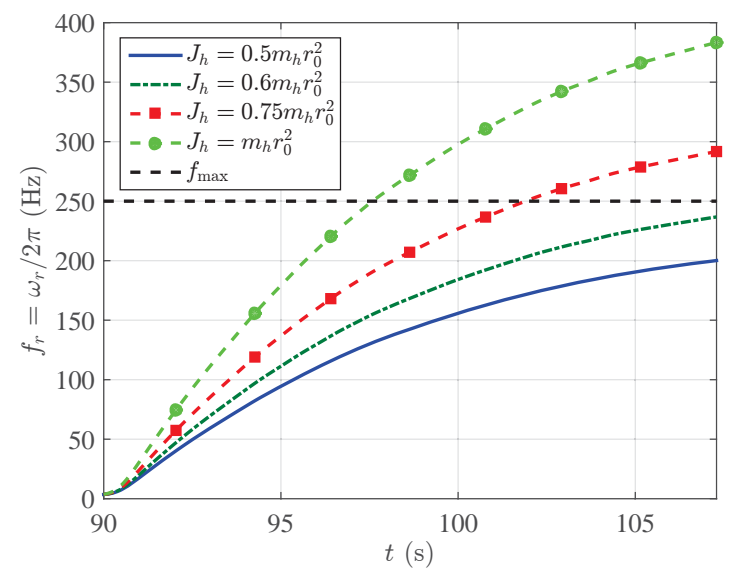

Fig. 12 Angular frequency of the reaction wheel before web deployment.

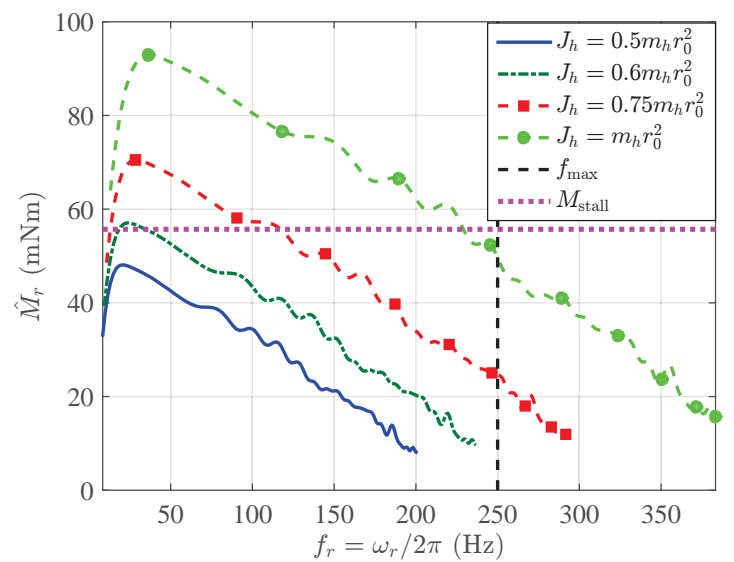

Fig. 13 Torque of the motor for the reaction wheel before web deployment. 
reaction wheel becomes

$$
J_{c}=\left(m_{\mathrm{hc}}+4 m_{d}+m_{\mathrm{web}}\right) r_{0}^{2}+0.5\left(m_{\mathrm{cp}}+m_{\mathrm{in}}\right) m r_{0}^{2}
$$

Input masses and radius in Eq. (17), we can get $J_{c}=42600 \mathrm{kgmm}^{2}$ which is a little less than $J_{c}=42800 \mathrm{kgmm}^{2}$ for $J_{h}=0.6 m_{h} r_{0}^{2}$ in Eq. (9) and agrees with the calculations from flight data through Figs. 12-13. Therefore $J_{h}=0.6 m_{h} r_{0}^{2}$ and the relationship between $\hat{M}_{r}$ and $\omega_{f}$ in Fig. 13 are used in following simulations.

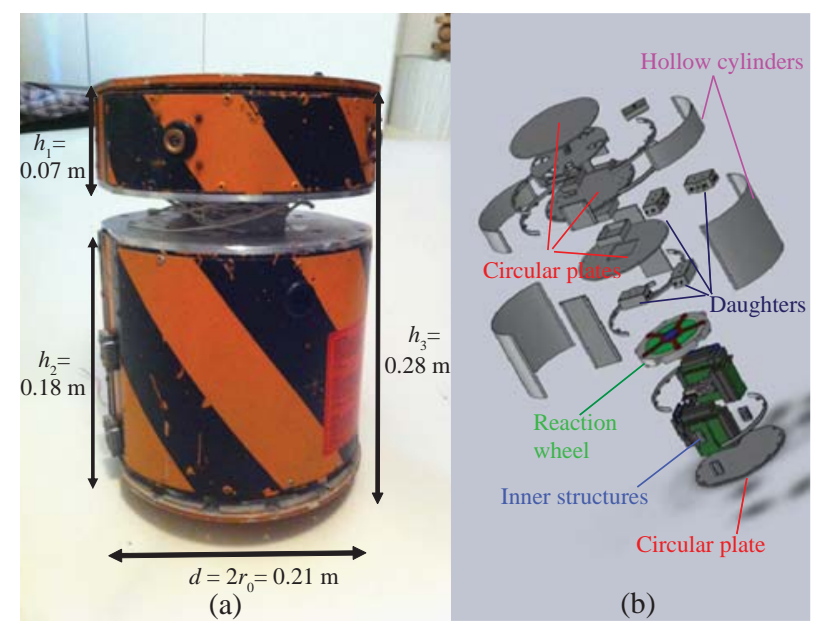

Fig. 14 (a) Assembled CHAD and (b) CAD model.

\subsection{Web deployment}

Since the IMU fixed in the center of CHAD did not function, the motion of the hub was not recorded. Fortunately, the deployment and stabilization phases were recorded by four cameras. Figure 15 shows the web in the deployment phase. The number in each photo represents the camera number around the hub with 90 degree separation in negative roll. The approximate angular frequency, Fig. 16, was calculated from the video footage according to the changing areas of Earth. For example, the area of Earth in camera 3 was increased from Fig. 15(a) to (c) but decreased from (d) to (f), which indicates that the hub changed direction between (b) and (e) during the deployment. This corresponds to the first point when the rotational direction changes in Fig. 16. 

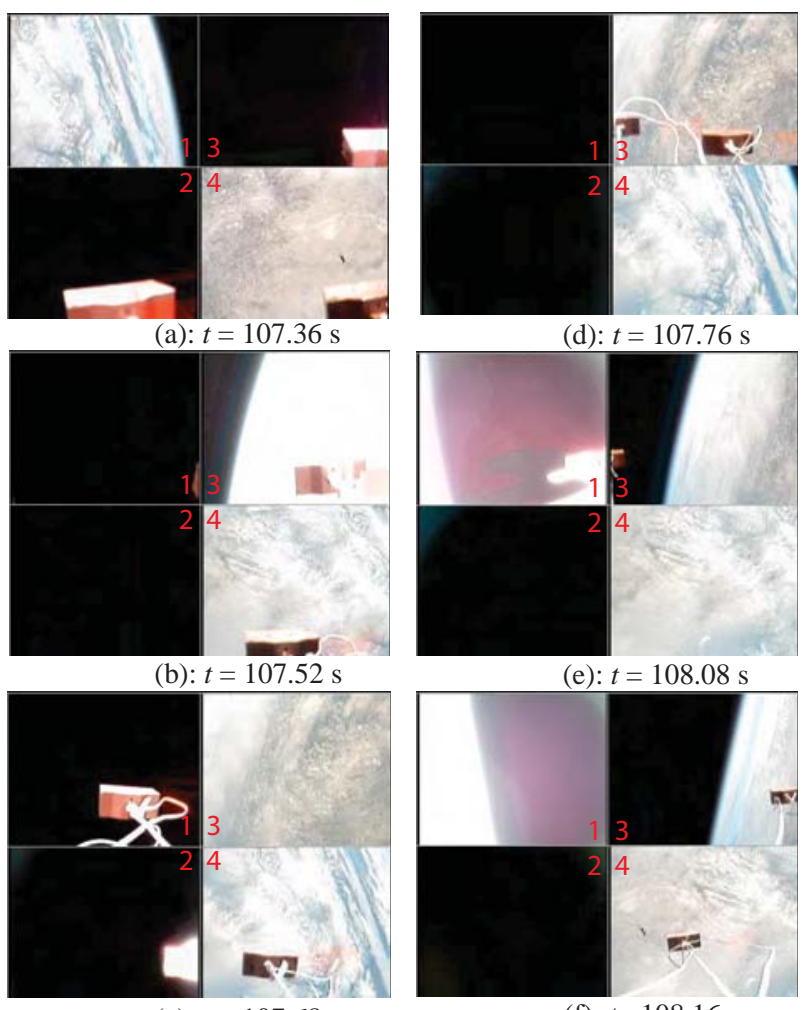

(c): $t=107.68 \mathrm{~s}$

(f): $t=108.16 \mathrm{~s}$

Fig. 15 Photos from the four cameras: web started to deploy.

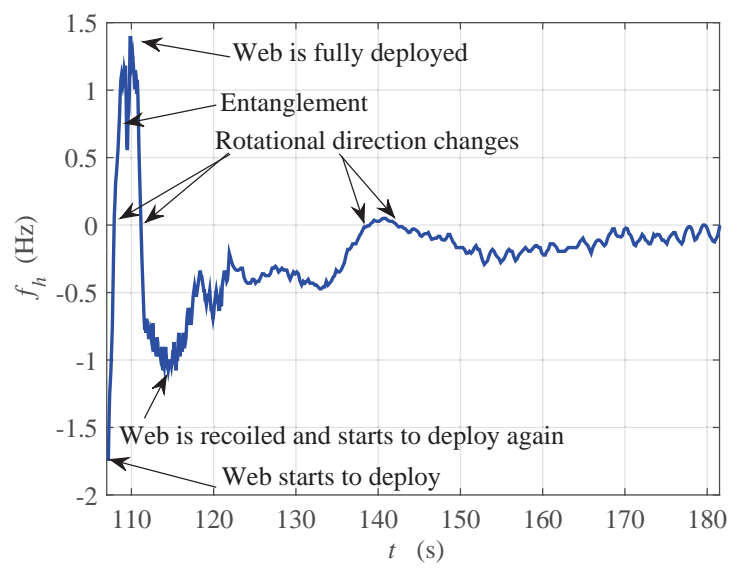

Fig. 16 Rotational frequency of the hub computed from video footage. 
In the beginning of deployment, only three daughters were shown in Fig. 15(a) and sometimes only two daughters in the cameras, Fig. 15(d)-(f). If there would be no large out-of-plane motion and if the web had deployed symmetrically, all four daughters would have be seen in the cameras. The out-of-plane motion can also be found from the acceleration of each IMU, Fig. 17, where $\mathrm{IMU}_{n}$ represents the IMU in the daughter section $n$, Fig. 1. The maximum out-of-plane acceleration $a_{z}$ was larger than $0.5 \mathrm{~g}$ in deployment phase. Figure 15(d) also shows two daughters were close to each other. One reason of that in-plane motion could be the friction force and Coriolis force as shown in ground test, Fig. 5(d), caused by the high deployment velocity and interaction between the web cords.

The magnitude of $a_{y}$ of $\mathrm{IMU}_{1}$ was a little smaller than the others, Fig. 18, which indicates an asymmetrical deployment due to the in-plane and out-ofplane motions. After the web recoiled and started to deploy again, acceleration $a_{y}$ of each IMU became different again. The numbers of peaks of the acceleration indicates that the web deployed and recoiled several times.

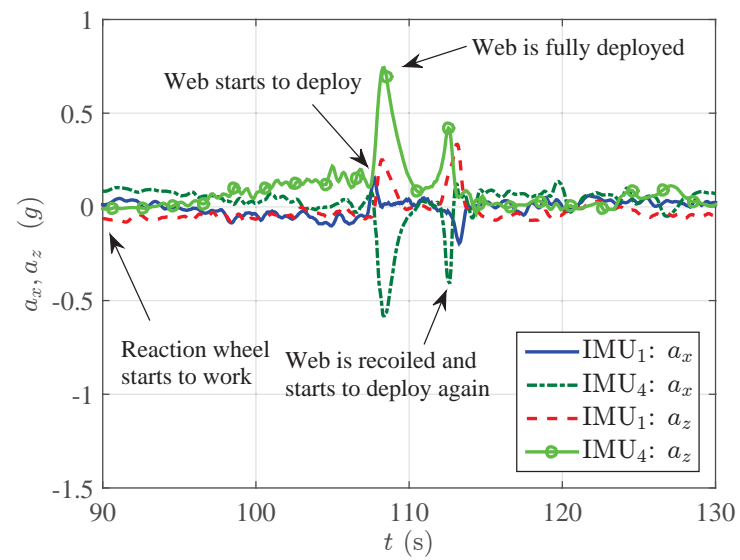

Fig. 17 Acceleration $a_{x}$ and $a_{z}$ from $\mathrm{IMU}_{1}$ and $\mathrm{IMU}_{4}$ after the reaction wheel started to work.

Figure 19(b) shows that a web arm was almost fully deployed, but before this, two daughters were entangled, Fig. 19(a). After full deployment, the web did not become stable but started to recoil, Fig. 19(d), and deployed again, Fig. 19(e). The too high initial angular velocity thus caused a too quick deployment and changed the rotational direction of the hub before the 


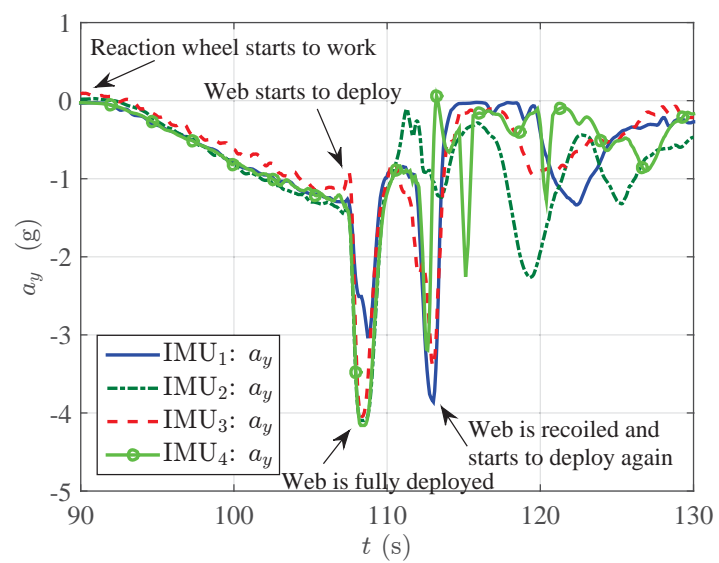

Fig. 18 Acceleration $a_{y}$ of each IMU after the reaction wheel started to work.

web was fully deployed and a too high angular velocity can also be found when the web was fully deployed, Fig. 16. An unstable deployment was observed in the video footage and confirmed by the accelerometers, Fig. 18. Four daughters were also not shown in any of the video footage in Fig. 19, which implies out-of-plane motions happened in the deployment and the angular velocities of daughters were not equal to each other or the hub. This is due to the slack, entanglements and asymmetry of the web deployment. However, since the cameras were above the plane of web deployment, the out-of-plane motions were not clearly captured.

\subsection{Simulation}

The question now is what would have happened if the controller had worked properly and the web had started to deploy at a proper initial angular velocity? In the following, we use our analytical model to reconstruct the deployment with $J_{h}=0.6 m_{h} r_{0}^{2}$ and the $\hat{M}_{r}$ from Fig. 13. Two different initial conditions were considered: the proper initial angular velocity $\omega_{0}=$ $-0.628 \mathrm{rad} / \mathrm{s}$ from design and the high amplitude angular velocity $\omega_{0}=$ $-11.3 \mathrm{rad} / \mathrm{s}$ from the actual experiment. Simulation results are shown in Fig. 20.

Before web deployment, the reaction wheel provided the maximum torque to the hub to reach the initial angular velocity. If the initial angular velocity of the CHAD is $-11.3 \mathrm{rad} / \mathrm{s}$ as in the actual experiment, the angular velocity of the reaction wheel would reach a very high spin rate from Eq. (13), and 


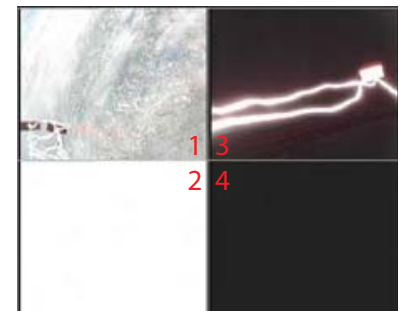

(a): $t=108.64 \mathrm{~s}$

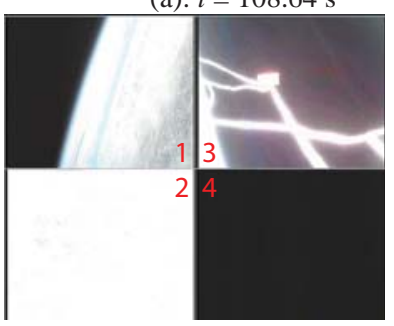

(b): $t=108.72$

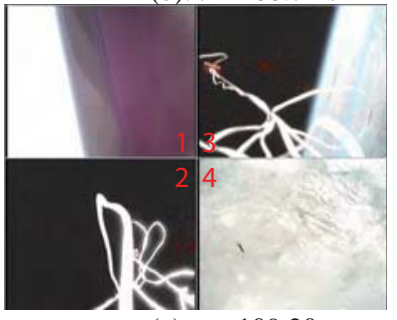

(c): $t=109.20 \mathrm{~s}$

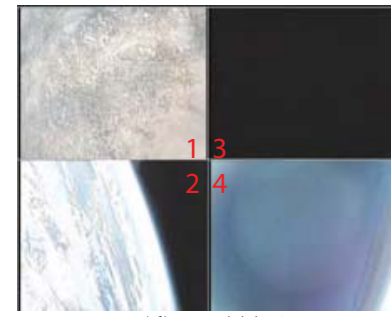

$(\mathrm{d}): t=111.6 \mathrm{~s}$

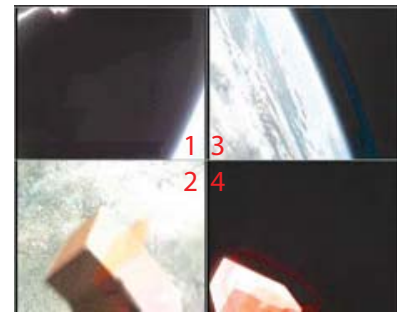

(e): $t=112.72 \mathrm{~s}$

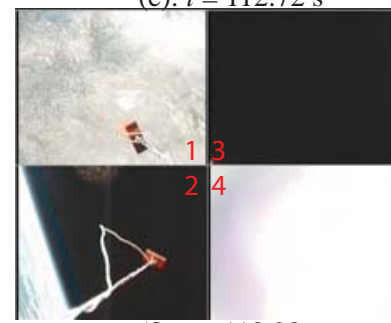

(f): $t=118.28 \mathrm{~s}$

Fig. 19 Photos from four cameras: (a)-(b), web was fully deployed; (c)-(d) recoiled; (e)-(f) deployed again. 
the maximum torque would decrease to around $10 \mathrm{mNm}$ according to Fig. 13 for $J_{h}=0.6 m_{h} r_{0}^{2}$. Therefore, the control torque is much lower for $\omega_{0}=$ $-11.3 \mathrm{rad} / \mathrm{s}$ than for $\omega_{0}=-0.628 \mathrm{rad} / \mathrm{s}$ and the motor cannot provide enough torque to stabilize the fast deployment, Fig. 20.

Comparing the angular velocity of the hub in the simulation and experiment when $\omega_{0}=-11.3 \mathrm{rad} / \mathrm{s}$, Fig. 21, the deployment time in simulation was faster than the experiment but reached to a similar maximum angular velocity at the first full deployment. In the simulation, the web keeps on deploying and recoiling since no energy dissipation is assumed. In reality, e.g. friction from interaction between the web and the hub would consume energy and delay deployment. In the actual deployment, entanglement were also observed, Fig. 19(a), which also consumed energy. When the web was fully deployed, shock forces and damping were another form of energy dissipation. After the web was fully deployed, the web started to recoil around the hub both in the simulations and in the experiment, Fig. 21.

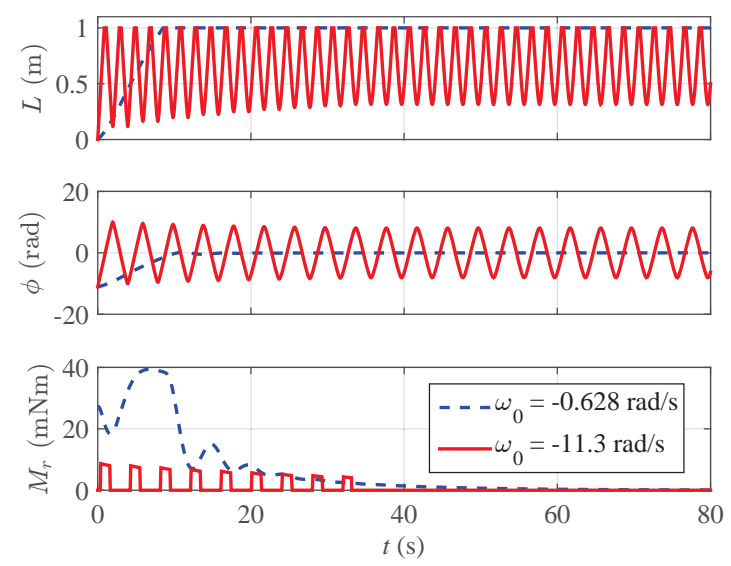

Fig. 20 Deployed length, $\phi$ and M.

The stability parameters $\gamma_{1}$ and $\gamma_{2}$ of a daughter according to Eqs. (1) and (2) are shown in Fig. 22 when $\omega_{0}=-0.628 \mathrm{rad} / \mathrm{s}$ was used in simulation. From 0 to $1 \mathrm{~s}, \gamma_{1}<1$, so the Coriolis force was higher than the centrifugal force. At $t=1 \mathrm{~s}$, the web was deployed only $0.08 \mathrm{~m}$, so the Coriolis force at this time would not have caused entanglement or unstable in-plane motion. After $t=1 \mathrm{~s}$, the centrifugal force was much higher than the Coriolis force, $\gamma_{1} \gg 1$. In comparison, $\gamma_{2}$ was kept at a very high magnitude from the 


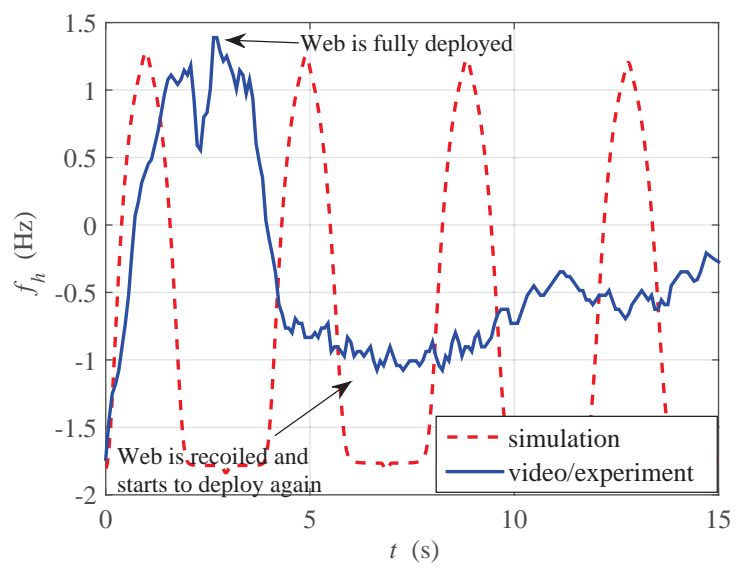

Fig. 21 Angular frequency of the hub in the simulation and video.

beginning of deployment which indicated that the inertial force was very small relative to the centrifugal force. Therefore, according to the simulation, if the deployment had started from the correct initial angular velocity, the motor used in the experiment most likely would be able to produce a stable deployment.

\section{Conclusion}

In this paper, the results of the Suaineadh web deployment experiment were analyzed and a simple analytical deployment model, used in the design, was used in the reconstruction of the actual flight results. Centrifugal forces deployed and stabilized the web and the motion was actively controlled by a reaction wheel. Scaled down ground tests provided evidence that if the angular velocity of the central hub was properly controlled, the web could be successfully deployed and stabilized. The proper initial and final angular velocities of the web deployment were inserted into the controller prior to launch. The web was designed to deploy after the rocket was de-spun to avoid a too high initial angular velocity. The folding pattern and coiling direction were decided based on the assumed rotational directions of the hub and the reaction wheel after ejection.

The experiment CHAD was spun up by the reaction wheel after ejection, the motor controller was activated and the daughters were released in 


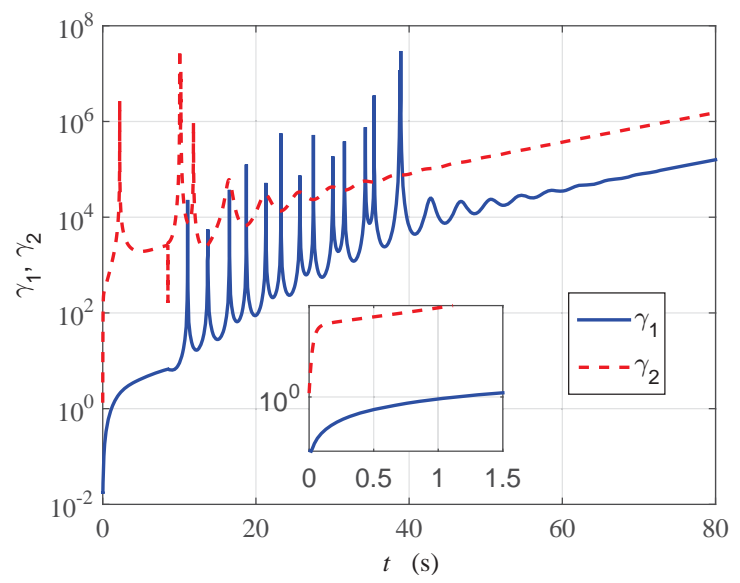

Fig. 22 The stability parameters $\gamma_{1}$ and $\gamma_{2}$ of a tip daughter when $\omega_{0}=-0.628 \mathrm{rad} / \mathrm{s}$ was used in simulation.

a milli-gravity environment. However, the web did not deploy at the designed specified initial angular velocity which might be due to an error that $\omega_{0}=0.628 \mathrm{rad} / \mathrm{s}$ was used instead of $\omega_{0}=-0.628 \mathrm{rad} / \mathrm{s}$ as the input in the controller, the hub sensor failed to detect the required spin rate of the hub, or other unknown problems. In order to avoid the input error in future similar experiments, a new "modified optimal MK control law" is proposed, Eq. (12). A time backup system was eventually activated and released the web, which led to a too high initial angular velocity and thus an uncontrollable deployment.

Some valuable data and experience were collected from the experiment. Data recorded in the IMUs and cameras were used to analyze the actual deployment behaviour and provided useful information about the system and deployment for simulation analysis. The approximate moment of inertia of the hub, $J_{h}$, and the maximum nonlinear torque of the motor, $\hat{M}_{r}$, were calculated from the data of the IMUs. We used simple simulation tools to reconstruct the unsuccessful deployment. The comparison between experiment results and simulations shows that high energy dissipation happened during recoiling. This might be caused by web slackening, damping, entanglement, friction and out-of-plane motions. The hub might also be wobbling during the deployment. It is difficult to conclude this because the sensor of the hub did not work, thus the wobbling motion was not measured. If the 
web had deployed at the proper initial angular velocity, the energy dissipation would be smaller and thus the simulation tools could well used to model the web deployment. Simulations indicate that the motor used in the experiment could provide enough torque for deployment and stabilization if the proper initial angular velocity had been reached, and a smaller momentum of inertia of the hub could make the deployment more stable. Parts were developed specifically for this experiment, including the reaction wheel, the web, the dedicated electronics and the control algorithm. The knowledge and experience gained from this sounding rocket experiment can be used for future similar missions.

It is noted that, this experiment is a student project on a short time schedule including students from many disciplines and universities in different countries, a number of companies and research organizations with a low budget. The major challenge is that so many requirements shall be verified in a short time schedule. If the controller was more thoroughly tested after the Suaineadh was assembled, the possible reason for the failure of the experiment might have been avoided. However, even with limited information and results, the deployment dynamics could be reconstructed thanks to video footage. Without the footage, it would not have been possible to perform the study in this paper.

\section{Acknowledgments}

The authors thank all the students in the two editions of the Suaineadh team: Andrew Feeney, Andrew Mathieson, Christie Maddock, Christopher Murray, Chris Unsworth, John Russell, Malcolm McRobb, Neil Smith, Norilmi Ismail and Paul Reynolds of University of Glasgow, Johannes Weppler of University of Strathclyde, Adam Wujek, Ali Dabiri, Carl Brengesjö, Fredrik Rogberg, Jerker Skogby, Junyi Wang, Martine Selin, Mengqi Zhang, Niklas Hansen, Pau Mallol and Rafael Ritterbusch of KTH and co-supervisors Professor Matthew Cartmell, Dr. Johnny Öberg and William Sandqvist. We also thank George Silvie of the University of Glasgow for the fabrication of the mechanical components, Dr. Giorgio Magistrati of ESTEC and Dr. Marco Molina of Carlo Gavazzi Space for help on thermal analysis. Special thanks go to Jerker Skogby and Adam Wujek for their help on the data analysis.

The financial support from the European Space Agency's Advanced Concepts Team and support from its members Leopold Summerer and Dario 
Izzo are greatly acknowledged. We also thank all people from DLR, SNSB, MORABA, SSC, Esrange and ESA Education Office involved in the REXUS8 campaign for continuous support and the launch opportunity. Finally, we acknowledge the financial support from KTH, University of Glasgow and University of Strathclyde.

\section{References}

[1] R. Freeland, G. Bilyeu, G. Veal, M. Steiner, D. Carson, Large inflatable deployable antenna flight experiment results, Acta Astronautica 41 (4) (1997) 267-277. doi:10.1016/S0094-5765(98)00057-5.

[2] A. Witkowski, G. Brown, Mars deployable decelerators capability roadmap summary, in: Aerospace Conference, 2006 IEEE, IEEE, Big Sky, Montana, 2006, p. 1585. doi:10.1109/AERO.2006.1655792.

[3] J. M. Fernandez, L. Visagie, M. Schenk, O. R. Stohlman, G. S. Aglietti, V. J. Lappas, S. Erb, Design and development of a gossamer sail system for deorbiting in low earth orbit, Acta Astronautica 103 (2014) 204-225. doi:10.1016/j.actaastro.2014.06.018.

[4] S. Nakasuka, T. Aoki, I. Ikeda, Y. Tsuda, Y. Kawakatsu, "Furoshiki Satellite" - a large membrane structure as a novel space system, Acta Astronautica 48 (5) (2001) 461-468. doi:10.1016/S0094-5765(01) 00056-X.

[5] N. Kaya, M. Iwashita, S. Nakasuka, L. Summerer, J. Mankins, Rocket experiment on construction of huge transmitting antenna for the SPS using Furoshiki satellite system with robots, in: Solar Power from SpaceSPS'04, 2004, pp. 231-235, ESA SP-567.

[6] N. Kaya, M. Iwashita, S. Nakasuka, L. Summerer, J. Mankins, Crawling robots on large web in rocket experiment on Furoshiki deployment, Journal of the British Interplanetary Society 58 (11-12) (2005) 403-406. doi:10.2514/6. IAC-04-R.1.07.

[7] S. Nakasuka, T. Funane, Y. Nakamura, Y. Nojiri, H. Sahara, F. Sasaki, N. Kaya, Sounding rocket flight experiment for demonstrating "Furoshiki Satellite" for large phased array antenna, Acta Astronautica 59 (1) (2006) 200-205. doi:10.1016/j . actaastro. 2006.02.014. 
[8] N. Kaya, M. Iwashita, K. Tanaka, S. Nakasuka, L. Summerer, Rocket experiment on microwave power transmission with Furoshiki deployment, Acta Astronautica 65 (1) (2009) 202-205. doi:10.2514/6. iac-06-c3. 3.03.

[9] V. Koshelev, V. Melnikov, Large space structures formed by centrifugal forces, Earth Space Institute Book Series, Gordon and Breach Science Publishers, Amsterdam, 1998.

[10] G. Tibert, M. Gärdsback, Space webs: final report, Noordwijk, The Netherlands, 2006, ESA Advanced Concepts Team, Rept. 05/4109a.

[11] M. Gärdsback, G. Tibert, Deployment control of spinning space webs, Journal of Guidance, Control and Dynamics 32 (1) (2009) 40-50. doi: 10.2514/1.37468.

[12] M. Gärdsback, G. Tibert, Optimal deployment control of spinning space webs and membranes, Journal of Guidance, Control and Dynamics 32 (5) (2009) 1519-1530. doi:10.2514/1.42203.

[13] H. U. Schuerch, R. MacNeal, Deployable centrifugally stabilized structures for atmospheric entry from space, National Aeronautics and Space Administration, USA, 1964, NASA contractor report 69.

[14] J. M. Hedgepeth, Dynamics of a large spin-stiffened deployable paraboloidal antenna, Journal of Spacecraft and Rockets 7 (9) (1970) 1043-1048. doi:10.2514/3.30100.

[15] V. Melnikov, K. Pichkhadze, Design of frameless SA deployed by centrifugal forces and its deployment mechanism as a basis of new technology of in-orbit power plant assembling, in: 56 th International Astronautical Congress, Vol. 6, International Astronautical Federation, American Institute of Aeronautics and Astronautics (AIAA), Paris, 2005, pp. 3972-3977. doi:10.2514/6. IAC-05-C2.P.01.

[16] Y. Tsuda, O. Mori, R. Funase, H. Sawada, T. Yamamoto, T. Saiki, T. Endo, J. Kawaguchi, Flight status of IKAROS deep space solar sail demonstrator, Acta Astronautica 69 (9-10) (2011) 833-840. doi:10. $1016 / j$. actaastro.2011.06.005. 
[17] M. Leipold, M. Eiden, C. Garner, L. Herbeck, D. Kassing, T. Niederstadt, T. Krüger, G. Pagel, M. Rezazad, H. Rozemeijer, W. Seboldt, C. Schöppinger, C. Sickinger, W. Unckenbold, Solar sail technology development and demonstration, Acta Astronautica 52 (2-6) (2003) 317326. doi:10.1016/s0094-5765(02)00171-6.

[18] J. Block, M. Straubel, M. Wiedemann, Ultralight deployable booms for solar sails and other large gossamer structures in space, Acta Astronautica 68 (7-8) (2011) 984-992. doi:10.1016/j.actaastro.2010.09.005.

[19] D. Haraguchi, H. Sakamoto, Y. Shirasawa, O. Mori, Design criteria for spin deployment of gossamer structures considering nutation dynamics, in: AIAA Guidance, Navigation, and Control Conference, Vol. 8072, American Institute of Aeronautics and Astronautics (AIAA), Toronto, Ontario, Canada, 2010. doi:10.2514/6.2010-8072.

[20] L. Johnson, R. Young, E. Montgomery, D. Alhorn, Status of solar sail technology within NASA, Advances in Space Research 48 (11) (2011) 1687-1694. doi:10.1016/j.asr.2010.12.011.

[21] Y. Yu, H. Baoyin, J. Li, Dynamic modelling and analysis of space webs, Science China Physics, Mechanics and Astronomy 54 (4) (2011) 783791. doi:10.1007/s11433-011-4290-4.

[22] N. Okuizumi, T. Yamamoto, Centrifugal deployment of membrane with spiral folding: experiment and simulation, Journal of Space Engineering 2 (1) (2009) 41-50. doi:10.1299/spacee.2.41.

[23] M. B. Quadrelli, J. West, Sensitivity studies of the deployment of a square inflatable solar sail with vanes, Acta Astronautica 65 (7-8) (2009) 1007-1027. doi:10.1016/j.actaastro.2009.03.015.

[24] M. Vasile, M. Cartmell, F. Zerihun Dejene, T. Drysdale, M. Alaniz Flores, M. Gulzar, N. Ismail, M. U. Khalid, M. Li, C. Maddock, P. Mallol, A. Mathieson, M. McRobb, J. Öberg, O. Purcell, P. Reynolds, R. Ritterbusch, W. Sandqvist, L. Summerer, M. U. Tanveer, G. Tibert, G. Whyte, W. Zafar, J. Zhang, The Suaineadh project: a stepping stone towards the deployment of large flexible structures in space, in: 61st International Astronautical Congress, IAC 2010, the International 
Astronautical Federation, Prague, Czech Republic, 2010, pp. IAC-10C3.4.

[25] T. Sinn, M. McRrobb, A. Wujek, J. Skogby, M. Zhang, M. Vasile, G. Tibert, J. Weppler, A. Feeney, J. Russell, REXUS 12 Suaineadh experiment: deployment of a web in microgravity conditions using centrifugal forces, in: 62nd International Astronautical Congress, no. IAC11-A2.3.7, Cape Town, South Africa, 2011, pp. 642-650.

[26] T. Sinn, M. McRobb, A. Wujek, J. Skogby, F. Rogberg, J. Wang, M. Vasile, G. Tibert, Lessons learned from REXUS12's Suaineadh experiment: spinning deployment of a space web in milli gravity, in: 21st ESA Symposium on European Rocket and Balloon Programmes and Related Research, Beijing, China, 2013, pp. 329-338.

[27] W. Nino, S. Patric, B. Wolfgang, F. Christopher, G. Patrick, P.-S. Kai, P. Anna, R. Matti, Z. Thomas, Alternative application of solar sail technology, in: Advances in Solar Sailing, Springer, 2014, pp. 351-365. doi:10.1007/978-3-642-34907-2_23. 